\title{
Front Matter: Volume 6629
}

, "Front Matter: Volume 6629," Proc. SPIE 6629, Diffuse Optical Imaging of Tissue, 662901 (20 July 2007); doi: 10.1117/12.754719

SPIE. Event: European Conferences on Biomedical Optics, 2007, Munich, Germany 


\title{
PROGRESS IN BIOMEDICAL OPTICS AND IMAGING
}

Vol. 8, No. 42

\section{Diffuse Optical Imaging of Tissue}

\author{
Brian W. Pogue \\ Rinaldo Cubeddu \\ Editors
}

19-21 June 2007

Munich, Germany

Sponsored and Published by

SPIE

OSA-Optical Society of America

Financial Sponsor

(9) EOARD—European Office of Aerospace Research and Development

We wish to thank the following for their contribution to the success of this conference:

European Office of Aerospace Research and Development

Air Force Office of Scientific Research

United States Air Force Research Laboratory (www.london.af.mil)

Cooperating Organizations

EOS-European Optical Society

WLT-Wissenschaftliche Gesellschaft Lasertechnik e.V. (Germany)

DGLM-Deutsche Gesellschaft für Lasermedizin e.V. (Germany) 
The papers included in this volume were part of the technical conference cited on the cover and title page. Papers were selected and subject to review by the editors and conference program committee. Some conference presentations may not be available for publication. The papers published in these proceedings reflect the work and thoughts of the authors and are published herein as submitted. The publisher is not responsible for the validity of the information or for any outcomes resulting from reliance thereon.

Please use the following format to cite material from this book:

Author(s), "Title of Paper," in Diffuse Optical Imaging of Tissue, edited by Brian W. Pogue, Rinaldo Cubeddu, Proceedings of SPIE-OSA Biomedical Optics Vol. 6629 (SPIE, Bellingham, WA, 2007) Article CID Number.

ISSN 1605-7422

ISBN 9780819467737

Copublished by

SPIE

P.O. Box 10, Bellingham, Washington 98227-0010 USA

Telephone +1 3606763290 (Pacific Time) · Fax +1 3606471445

SPIE.org

and

OSA-Optical Society of America

2010 Massachusetts Ave., N.W., Washington, D.C., 20036 USA

Telephone +1 2022238130 (Eastern Time) · Fax +1 2022231096

osa.org

Copyright (c) 2007, Society of Photo-Optical Instrumentation Engineers and Optical Society of America.

Copying of material in this book for internal or personal use, or for the internal or personal use of specific clients, beyond the fair use provisions granted by the U.S. Copyright Law is authorized by the publishers subject to payment of copying fees. The Transactional Reporting Service base fee for this volume is $\$ 18.00$ per article (or portion thereof), which should be paid directly to the Copyright Clearance Center (CCC), 222 Rosewood Drive, Danvers, MA 01923. Payment may also be made electronically through CCC Online at copyright.com. Other copying for republication, resale, advertising or promotion, or any form of systematic or multiple reproduction of any material in this book is prohibited except with permission in writing from the publisher. The CCC fee code is $1605-7422 / 07 / \$ 18.00$.

Printed in the United States of America.

Paper Numbering: Proceedings of SPIE-OSA Biomedical Optics follow an e-First publication model, with papers published first online and then in print and on CD-ROM. Papers are published as they are submitted and meet publication criteria. A unique, consistent, permanent citation identifier (CID) number is assigned to each article at the time of the first publication. Utilization of CIDs allows articles to be fully citable as soon they are published online, and connects the same identifier to all online, print, and electronic versions of the publication. In this six-digit CID article numbering system:

- The first four digits correspond to the volume number.

- The last two digits indicate publication order within the volume using a Base 36 numbering system employing both numerals and letters. These two-number sets start with $00,01,02,03,04$, $05,06,07,08,09,0 A, 0 B \ldots 0 Z$, followed by 10-1Z, 20-2Z, etc.

The CID number appears on each page of the manuscript. The complete citation is used on the first page, and an abbreviated version on subsequent pages. Numbers in the index correspond to the last two digits of the six-digit CID number. 


\section{Contents}

ix Conference Committee

\section{SESSION 1 NEW TECHNOLOGIES}

662902 Fluorescence diffuse optical tomography: a wavelet-based model reduction [6629-01]

A. Frassati, A. DaSilva, J.-M. Dinten, LETI-CEA-MINATEC Recherche Technologique (France);

D. Georges, GIPSA-Lab. (France)

662903 Dynamic optical tomographic imager with optimized digital lock-in filtering [6629-02] J. M. Lasker, J. M. Masciotti, Y. Li, C. Fong, A. H. Hielscher, Columbia Univ. (USA)

662904 Speckle pattern characterization by circular statistics [6629-03]

M. C. Péron, E. Deléchelle, Lab. Images Signaux et Systèmes Intelligents (France); S. Guyot, Lab. de Physique des Interfaces et des Couches Minces, École Polytechnique (France)

\section{SESSION 2 IMAGE RECONSTRUCTION}

662906 Evaluation of image reconstruction algorithm for near infrared topography by virtual head phantom (Invited Paper) [6629-05]

H. Kawaguchi, E. Okada, Keio Univ. (Japan)

662907 Near-surface sensitivity suppression way for diffuse reflective optical tomography: simulation and a phantom study [6629-06]

K. Fukuda, Tokyo Metropolitan College of Industrial Technology (Japan); M. Fujii, Sophia Univ. (Japan)

662908 Novel method for depth-resolved brain functional imaging by time-domain NIRS [6629-07]

D. Contini, L. Spinelli, A. Torricelli, A. Pifferi, R. Cubeddu, ULTRAS-INFM-CNR and IFN-CNR, Politecnico di Milano (Italy)

6629 OA Wavelengths optimization in multispectral diffuse optical tomography considering uncertainties in absorption spectra [6629-09]

B. Brendel, T. Nielsen, Philips Research Europe, Hamburg (Germany)

6629 OC Depth selective diffuse optical computed topography: simulations and phantom experiments [6629-11]

M. Fujii, A. Kawanaka, Sophia Univ. (Japan); K. Nakayama, Electo-Design Company Ltd. (Japan) 
6629 OD Assessment of collagen absorption and related potential diagnostic applications (Invited Paper) [6629-12]

P. Taroni, D. Comelli, A. Giusto, A. Pifferi, ULTRAS-CNR-INFM and IFN-CNR, Politecnico di Milano (Italy); N. Shah, Beckman Laser Institute (USA); L. Spinelli, A. Torricelli, R. Cubeddu, ULTRAS-CNR-INFM and IFN-CNR, Politecnico di Milano (Italy)

6629 OE Influence of cell shape and orientation on the optical properties of human erythrocytes [6629-13]

M. Meinke, Charité-Universitätsmedizin Berlin (Germany); M. Friebel, Laser- und MedizinTechnologie GmbH (Germany); G. Müller, Charité-Universitätsmedizin Berlin (Germany)

6629 OF Detection and characterization of an optical inhomogeneity by diffuse photon-pairs density wave in a multiple-scattering medium [6629-14]

L.-P. Yu, National Yang Ming Univ. (Taiwan); J.-S. Wu, National Central Univ. (Taiwan); Y.-H. Chan, Chang Gung Memorial Hospital, Chang Gung Univ. College of Medicine (Taiwan); L.-C. Su, National Central Univ. (Taiwan); C. Chou, National Yang Ming Univ. (Taiwan) and National Central Univ. (Taiwan)

6629 OG Depth resolution by continuous-wave imaging [6629-15]

E. B. Aksel, A. Akin, Bogaziçi Univ. (Turkey)

$6629 \mathrm{OH} \quad \mathrm{CW}$ and time domain procedures for accurate calibration of optical properties of liquid diffusive media at NIR wavelengths [6629-16]

F. Martelli, Univ. degli Studi di Firenze (Italy); L. Spinelli, A. Farina, A. Pifferi, A. Torricelli, R. Cubeddu, ULTRAS-CNR-INFM and IFN-CNR, Politecnico di Milano (Italy); G. Zaccanti, Univ. degli Studi di Firenze (Italy)

662901 Determination of the optical properties of turbid media by measurement of the spatially resolved reflectance [6629-17]

M. Pilz, A. Kienle, Institut für Lasertechnologien in der Medizin und Meßtechnik (Germany)

6629 0J Light attenuation through turbid slabs calculated by solutions of the Maxwell equations [6629-18]

J. Schäfer, A. Kienle, F. K. Forster, Institut für Lasertechnologien in der Medizin und Meßtechnik (Germany); A. Strey, Univ. Ulm (Germany)

6629 OK Path-length correction for the haemoglobin-concentration measurement using the skull cranial window by multi-spectral imaging analysis [6629-19]

K. Sakaguchi, S. Furukawa, Keio Univ. (Japan); T. Katsura, K. Yamazaki, H. Kawaguchi, A. Maki, Hitachi, Ltd. (Japan); E. Okada, Keio Univ. (Japan)

$6629 \mathrm{OL}$ Time-resolved measurement of the scattered light with an interferometric method based on the use of a camera [6629-20]

D. Ettori, K. Zarychta, E. Tinet, S. Avrillier, J.-M. Tualle, Lab. de Physique des Lasers, CNRS, Univ. Paris 13 (France) 
6629 OM Determination of the optical properties of anisotropic biological media using isotropic and anisotropic diffusion models [6629-21]

A. Kienle, C. Wetzel, Institut für Lasertechnologien in der Medizin und Meßtechnik (Germany); A. Bassi, D. Comelli, P. Taroni, A. Pifferi, Politecnico di Milano, ULTRAS-CNR-INFM and IFN-CNR (Italy)

\section{SESSION 4 MUSCLE AND VASCULAR IMAGING}

6629 ON Imaging of hemodynamic effects in arthritic joints with dynamic optical tomography (Invited Paper) [6629-22]

A. H. Hielscher, J. M. Lasker, C. J. Fong, E. Dwyer, Columbia Univ. (USA)

6629 OP Algorithms for muscle oxygenation monitoring corrected for adipose tissue thickness [6629-25]

D. Geraskin, Univ. of Applied Sciences Koblenz (Germany); P. Platen, J. Franke, Ruhr Univ. Bochum (Germany); M. Kohl-Bareis, Univ. of Applied Sciences Koblenz (Germany)

\section{SESSION 5 BRAIN IMAGING}

6629 OS Modeling of influence of frontal sinus on NIRS signal of brain activation [6629-28]

D. Yamamoto, S. Kuroda, E. Okada, Keio Univ. (Japan)

6629 OT Optical tomographic imaging of activation of the infant auditory cortex using perturbation Monte Carlo with anatomical a priori information [6629-29]

J. Heiskala, Helsinki Univ. Central Hospital (Finland), Helsinki Univ. of Technology (Finland), and Univ. of Helsinki (Finland); K. Kotilahti, L. Lipiäinen, P. Hiltunen, Helsinki Univ. of Technology (Finland) and Univ. of Helsinki (Finland); P. E. Grant, Massachusetts General Hospital (USA); I. Nissilä, Helsinki Univ. of Technology (Finland) and Univ. of Helsinki (Finland)

6629 OU Cerebral oxygenation monitoring during cardiac bypass surgery in infants with broad band spatially resolved spectroscopy [6629-30]

J. Soschinski, Univ. of Applied Sciences Koblenz (Germany); L. Ben Mine, Cologne Univ. (Germany); D. Geraskin, Univ. of Applied Sciences Koblenz (Germany); G. Bennink,

Cologne Univ. (Germany); M. Kohl-Bareis, Univ. of Applied Sciences Koblenz (Germany)

6629 OW Time-resolved non-contact fluorescence diffuse optical tomography measurements with ultra-fast time-correlated single photon counting avalanche photodiodes [6629-32]

Y. Bérubé-Lauzière, V. Robichaud, É. Lapointe, Univ. de Sherbrooke (Canada)

\section{SESSION 6 FLUORESCENCE IMAGING}

6629 OY Time-of-flight non-contact fluorescence diffuse optical tomography with numerical constant fraction discrimination (Invited Paper) [6629-34]

Y. Bérubé-Lauzière, V. Robichaud, Univ. de Sherbrooke (Canada) 
$662910360^{\circ}$ free space fluorescence molecular tomography using silhovette surface reconstruction [6629-36]

T. Lasser, Technical Univ. Munich (Germany); N. Deliolanis, A. Soubret, Massachussetts General Hospital and Harvard Medical School (USA); J. Ripoll, Foundation for Research and Technology Hellas (Greece); V. Ntziachristos, Massachussetts General Hospital and Harvard Medical School (USA)

662911 Whole body in vivo examination of small animals by simultaneous X-rays/optical tomography: comparison between the reconstructions obtained with different types of fluorescent labels [6629-37]

A. Da Silva, T. Bordy, M. Debourdeau, J.-M. Dinten, P. Peltié, P. Rizo, LETI-CEA MINATEC (France)

662912 Diffuse optical tomography forward model refinements in media with heterogeneous optical properties [6629-38]

S. Fortier, F. Leblond, ART Advanced Research Technologies Inc. (Canada)

\section{SESSION 7 BREAST IMAGING}

662915 fDOT for in vivo follow-up of tumor development in mice lungs (Invited Paper) [6629-41]

A. Koenig, L. Hervé, A. Da Silva, J.-M. Dinten, J. Boutet, M. Berger, LETI-CEA MINATEC (France); V. Josserand, Animage (France); J. Coll, INSERM, Institut Albert Bonniot (France); P. Peltié, P. Rizo, LETI-CEA MINATEC (France)

662917 First clinical trials of the Twente photoacoustic mammoscope (PAM) [6629-43] S. E. Vaartjes, J. C. G. van Hespen, Univ. of Twente (Netherlands); J. M. Klaase, F. M. van den Engh, A. K. H. Thé, Medisch Spectrum Twente (Netherlands); W. Steenbergen, T. G. van Leeuwen, S. Manohar, Univ. of Twente (Netherlands)

6629 1C Radiotherapy dosimetry assessment with optical projection tomography [6629-48] G. Zacharakis, Foundation for Research and Technology Hellas (Greece); A. Papadakis, F. Zacharopoulou, Univ. Hospital of Heraklion, Univ. of Crete (Greece); A. Garofalakis, Foundation for Research and Technology Hellas (Greece); T. Maris, Univ. Hospital of Heraklion, Univ. of Crete (Greece); J. Ripoll, Foundation for Research and Technology Hellas (Greece)

\section{POSTER SESSION}

$6629 \mathrm{lF}$ High frequency oscillations in brain hemodynamic response [6629-50]

A. Akin, Boĝaziçi Univ. (Turkey); H. Bolay, Gazi Univ. (Turkey)

6629 1G Analysis of skin recovery from mechanical indentation using diffuse lighting and digital imaging [6629-51]

N. T. Clancy, M. J. Leahy, Univ. of Limerick (Ireland); G. E. Nilsson, C. Anderson, Linköpings Univ. (Sweden)

$6629 \mathrm{1H} \quad$ Filtering effect to improve the reconstructed image quality of diffuse optical imaging [6629-52]

M.-C. Pan, Tung-Nan Institute of Technology (Taiwan); C.-H. Chen, L.-Y. Chen, M.-C. Pan, National Central Univ. (Taiwan) 
$66291 \mathrm{~J}$ Development of a computer vision binocular system for non-contact small animal model skin cancer tumour imaging [6629-54]

D. S. Gorpas, K. Politopoulos, D. Yova, National Technical Univ. of Athens (Greece)

$66291 \mathrm{~K}$ Three dimensional near infrared tomography of the breast [6629-56]

M. E. Eames, Univ. of Exeter (United Kingdom); B. W. Pogue, C. M. Carpenter, Dartmouth College (USA); H. Dehghani, Univ. of Exeter (United Kingdom)

$66291 \mathrm{~L} \quad$ Monitoring muscle metabolic indexes by time-domain near infrared spectroscopy during knee flex-extension induced by functional electrical stimulation [6629-57]

A. Torricelli, D. Contini, L. Spinelli, R. Cubeddu, ULTRAS-INFM-CNR and IFN-CNR, Politecnico di Milano (Italy); F. Molteni, Ctr. di riabilitazione Villa Beretta (Italy); S. Ferrante,

A. Pedrocchi, G. Ferrigno, NITLAB-TBMLAB, Politecnico di Milano (Italy)

$66291 \mathrm{M}$ Continuous performance test assessed with time-domain functional near infrared spectroscopy [6629-58]

A. Torricelli, D. Contini, L. Spinelli, M. Caffini, ULTRAS-INFM-CNR and IFN-CNR, Politecnico di Milano (Italy); M. Butti, G. Baselli, A. M. Bianchi, Politecnico di Milano (Italy); A. Bardoni, IRCCS E. Medea (Italy); S. Cerutti, Politecnico di Milano (Italy); R. Cubeddu, ULTRAS-INFMCNR and IFN-CNR, Politecnico di Milano (Italy)

$66291 \mathrm{~N}$ Measurement of the phase function of phantom medias with a two axis goniometer [6629-59]

R. Michels, S. Boll, A. Kienle, Institut für Lasertechnologien in der Medizin und Meßtechnik (Germany)

6629 IP Spatial resolved diffuse reflection as a tool for determination of size and embedding depth of blood vessels [6629-61]

A. V. Bykov, Univ. of Oulu (Finland) and M.V. Lomonosov Moscow State Univ. (Russia); A. V. Priezzhev, M.V. Lomonosov Moscow State Univ. (Russia); R. Myllyla, Univ. of Oulu (Finland)

$66291 Q$ Sensitivity analysis for small imaging domains using the frequency-domain transport equation [6629-62]

X. GU, K. Ren, A. H. Hielscher, Columbia Univ. (USA)

6629 1R Transmission RF diffuse optical tomography instrument for human breast imaging [6629-63] K. Lee, S. D. Konecky, R. Choe, H. Y. Ban, A. Corlu, T. Durduran, A. G. Yodh, Univ. of Pennsylvania (USA)

$66291 \mathrm{U}$ Approach to estimating low contrast inclusion with a priori guidance [6629-66] M.-C. Pan, C.-H. Chen, L.-Y. Chen, National Central Univ. (Taiwan); M.-C. Pan, Tung-Nan Institute of Technology (Taiwan)

$66291 \mathrm{~V} \quad$ Fluorescence lifetime imaging through turbid media reconstructed in the Fourier domain using time-gated imaging data [6629-67]

V. Y. Soloviev, Univ. College London (United Kingdom); K. B. Tahir, J. McGinty, D. S. Elson, M. Neil, Imperial College London (United Kingdom); A. Sardini, J. Hajnal, Imperial College Faculty of Medicine (United Kingdom); S. R. Arridge, Univ. College London (United Kingdom); P. M. W. French, Imperial College London (United Kingdom) 
6629 IW Imaging through a biological medium using speckle noise removal techniques [6629-68]

A. Cuddihy, B. Hennelly, National Univ. of Ireland Maynooth (Ireland); T. J. Naughton,

National Univ. of Ireland Maynooth (Ireland) and Univ. of Oulu (Finland); C. Markham,

R. O'Neill, National Univ. of Ireland Maynooth (Ireland)

Author Index 


\title{
Conference Committee
}

\author{
General Chairs
}

David Boas, Massachusetts General Hospital (USA)

Stefan Andersson-Engles, Lunds Tekniska Högskola (Sweden)

Program Chairs

Wolfgang Drexler, Cardiff University (United Kingdom)

Mary-Ann Mycek, University of Michigan (USA)

\section{Conference Chairs}

Brian W. Pogue, Dartmouth College (USA)

Rinaldo Cubeddu, ULTRAS-INFM-CNR and IFN-CNR, Politecnico di Milano (Italy)

Program Committee

Simon R. Arridge, University College London (United Kingdom)

Hamid Dehghani, The University of Exeter (United Kingdom)

Andreas H. Hielscher, Columbia University (USA)

Rainer Macdonald, Physikalisch-Technische Bundesanstalt (Germany)

Eiji Okada, Keio University (Japan)

Henricus J. C. M. Sterenborg, University of Rotterdam (Netherlands)

Jean-Michel Tualle, Laboratoire de Physique des Lasers, CNRS, Universitè Paris 13 (France)

\section{Session Chairs}

1 New Technologies

Andreas H. Hielscher, Columbia University (USA)

2 Image Reconstruction

Hamid Dehghani, University of Exeter (United Kingdom)

3 Tissue Optical Properties

Jean-Michel Tualle, Laboratoire de Physique des Lasers, CNRS,

Universitè Paris 13 (France)

$4 \quad$ Muscle and Vascular Imaging

Maurice C. G. Aalders, Universiteit van Amsterdam (Netherlands) 
$5 \quad$ Brain Imaging

Eiji Okada, Keio University (Japan)

Rainer Macdonald, Physikalisch-Technische Bundesanstalt (Germany)

6 Fluorescence Imaging

Rinaldo Cubeddu, Politecnico di Milano (Italy)

$7 \quad$ Breast Imaging

Brian W. Pogue, Dartmouth College (USA)

Poster Session

Rinaldo Cubeddu, ULTRAS-INFM-CNR and IFN-CNR, Politecnico di Milano (Italy) 